\title{
Social Environment As A Determinant Of Coping Self-Efficacy In Men And Women Living With HIV Under The Auspice Of A Large Healthcare Provider In Kenya
}

\author{
Thomas Matenjwa Kamau, Ohio University, USA \\ Valerie G. Olson, Dominican College, USA \\ Genevieve Pinto Zipp, Seton Hall University, USA \\ MaryAnn Clark, Long Island University, USA
}

\begin{abstract}
Kenya faces a severe, generalized HIV epidemic that continues to have a devastating impact on men and women living with HIV and, generally, all sectors of the population. HIV-related stress based on living with HIV, fear of progression of HIV to AIDS or to death, stigma, and discrimination among others are constant stressors afflicting people living with HIV. There are two ways to help people face these stressors - changing the internal characteristics of a person or changing the social environment.
\end{abstract}

A major healthcare provider engages a therapeutic coping model based on stress, appraisal, and coping theory that aims not only directly at changing internal dynamics of the person, but also changing these dynamics by altering the environment of men and women living with HIV. The interplay of a person's effort with a socially supportive environment is believed to foster coping self-efficacy (CSE). However, the efficacy of this therapeutic model has not been assessed and documented.

The purpose of this study was to determine the role of the social environment in shaping CSE among men and women living with HIV enrolled in the treatment and care program under the auspices of a large healthcare provider in Kenya. Three types of social support are: 1) emotional, which reduces anxiety and promotes self-esteem; 2) informational, which provides needed knowledge and skills useful in solving problems; and 3) belonging, which helps to distract a distressed person from disturbing thoughts significantly predicted CSE. The results support interventions that strengthen a person's character while encouraging family members, friends, and other social ties to act and react favorably, thereby creating a supportive environment. Implications, limitations, and future directions are discussed.

Keywords: HIV; Coping Self-Efficacy; Social Support; Dimensions of Social Support

\section{INTRODUCTION}

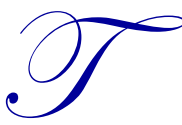

here are multiple HIV-related events that affect the welfare of people living with HIV. In the United States, Siegel and Scrimshaw (2005) studied a matched sample of women living with HIV before the advent of HIV medication and compared them with a sample of women who were prescribed antiretroviral therapy (ART). The researchers found that women on ART were significantly more likely than HIV- 
positive women who lived in the pre-ART era to report problems associated with being HIV positive. They were more likely to report HIV-related stress, experiences of stigma and that they would not seek solutions to the triggers of their stressful experience (avoidant coping). Literature also indicates that the diagnosis of HIV often leads to fear of disclosure of HIV status, discrimination and isolation, stress over complex treatment regimens, fear of side effects from medication, fear of disease progression to full-blown AIDS, and anxiety about dying (Catz et al., 2000; Theuninck, Lake, \& Gibson, 2010).

In Kenya, increased access to HIV medication partly solves the larger problem among people living with HIV, which means that other resources are still needed to address their needs. The results of the Kenya Demographic and Health Survey (KDHS) of 2004 revealed that over $40 \%$ of women and $26 \%$ of men in Kenya will not buy fresh vegetables from a vendor who is HIV positive (Central Bureau of Statistics [CBS], 2004). Furthermore, $43 \%$ of men and $40 \%$ of women in Kenya believe that a female teacher who is HIV positive should not be allowed to continue to teach. The inability to deal with these physical, emotional, and interpersonal stressors may further impact the lives of HIV-positive individuals and result in the development of secondary illnesses (Cohen, Janicki-Deverts, \& Miller, 2007; Leserman et al., 2002). To sum up, individuals living with HIV face several different HIV stressors and require coping resources to combat them.

Access to coping resources may explain variability in responses among people confronted by similar negative events. This stressor response variability can partly be attributed to differences in individuals' cognitive appraisal. The appraisals consist of the processes that mediate between the encounter and the reaction, such that some individuals may judge themselves incapable where others would judge themselves capable of tackling the same problems (Lazarus \& Folkman, 1984). Individuals who judge themselves as incapable may not have the motivation to take action, and those who judge themselves capable may actively engage in actions that can reduce or eliminate the perturbing issue(s) (Lazarus \& Folkman, 1984). The belief in one's capabilities to produce a desired outcome despite difficulties - termed coping self-efficacy (CSE) - may determine whether individuals will take action.

CSE refers to beliefs in one's capabilities to regulate emotional states and organize and execute the courses of action required to manage prospective situations, as well as to deal with the barriers that arise in order to ensure sustained action (Bandura, 1997; Chesney, Neilands, Chambers, Taylor, \& Folkman, 2006; Schwarzer \& Renner, 2000). Chesney et al. (2006) suggest that coping efforts may be emotion-focused, problem-focused, or a reappraisal of an event. Emotion-focused coping includes minimizing, avoiding, tolerating, and accepting stressful conditions and problem-focused coping exerts an effort directed at managing the problem. With new information, individuals may reappraise a situation, which may result in taking new approaches or adapting to the problem, particularly if nothing else can be done. Ultimately, whether people can perform specific tasks depends upon their confidence in their capability to carry out coping actions (Bandura, 1997); hence, the construct of CSE.

But how does one develop his or her belief of self-efficacy? Bandura (1997) postulated that efficacy beliefs are constructed through vicarious experiences and verbal persuasions, among others. Vicarious experiences - seeing people similar to one's self fight life stressors - may successfully bolster CSE in the observer's confidence that he or she possesses the capabilities to master comparable activities. Also, self-efficacy beliefs are reinforced through verbal persuasions, particularly by confidants or significant others. Theoretically, whether CSE is constructed vicariously or persuasively, it requires social ties - a network of people - through which individuals observes others similar to his or herself successfully fighting stressors.

Cohen and Wills (1985) posit a theory that social support fosters individuals' coping with stressful events, thus protecting them from potential bad effects of stressors. However, because the concept of social support is overly inclusive, it is important to understand the interrelatedness and the distinction between social structures and quality of social ties, all of which are considered to describe social support. Although a structure of people is necessary to generate an atmosphere of helpfulness, it does not necessarily produce the support that is needed. The quality of a tie is equally important because it determines its ability to provide coping resources. As Langford, Bowsher, Maloney, and Lillies (1997) argue, not only do individuals under stressful events need a structure of people, but, more importantly, the structure needs to generate the needed social resources. Although the concept of CSE could be construed as an individual trait, it is also a social environmental issue because an individual's 
psychological disposition is also influenced by the environment (Cohen \& Wills, 1985). Personal variables, such as commitments and belief or sense of control, are not innate but likely occur because of both the individual's characteristics and the social environment (Bandura, 1997). Social resources are particularly critical to individuals when they appraise a situation as taxing and exceeding their resources (Lazarus \& Folkman, 1984). To that end, interventions aimed at improving CSE would be effective if they were directed at changing the individual and the social environment. These changes may be necessary for individuals who had judged themselves incapable of coping with stressors or whose environment failed to generate resources. For instance, because of past enacted stigma (discrimination) against people living with HIV, such individuals tend to harbor distrust and thus decline to seek social support from the community, family, and even friends. They should be able to perceive that support is available if and when needed before they decide to seek it.

Researchers have defined perceived social support as the support resources perceived by people to be available if and when needed (Cohen \& Hoberman, 1983; Hutchinson, 1999). These support resources are classified as emotional, which reduce anxiety and foster self-esteem; informational, which provide critical knowledge and skills; material, which are useful in solving practical problems; and belonging, which help distract a distressed person from disturbing thoughts. Cohen and Wills (1985) argue that all these types of social support are resources that people under stress can leverage to cope with life stressors.

In Kenya, several interventions to reduce stress in people infected with HIV are being implemented. Nazareth Hospital in Nairobi, Kenya, has initiated one of the largest and comprehensive HIV-treatment and care programs, with over 4,000 men and women living with HIV enrolled. This program aims to foster positive thinking in individuals living with HIV to strengthen their beliefs in their abilities to fight stress. However, in recognition that actions are performed in a social context, the program also exerts efforts to change the environment in which clients, family, friends, and social groups are trained to support people living with HIV. The designers of the intervention believe that such an intervention will lead to the acquisition of skills and thus raise individuals' beliefs in their capabilities to regulate distress and manage problems as they unfold.

The program incorporates psychological and social interventions, such as individual and group counseling, training for adherence self-efficacy or CSE, provision of material support and relevant information, and assigning new patients' confidants to enhance their capability to deal with acute life events and dealing with HIV. However, the effectiveness of these interventions in increasing CSE in Kenyans living with HIV is limited, which makes it difficult to tell whether these programs are successful in meeting their goals. The purpose of the study was to test two hypotheses or address two research questions: 1) Whether there is a significant relationship between perceived social support and CSE and 2) whether there is a significant relationship between the dimensions of social support and CSE in Kenyans living with HIV.

\section{METHODS}

This was a cross-sectional study design. Several permissions were sought and obtained to conduct the study. Participants were recruited through flyers displayed in physicians' and counselor's waiting rooms and in the pharmacy department. Four research assistants were recruited and trained on how to recruit subjects, to obtain their informed consent, and to protect human subjects. Each research assistant also successfully completed the National Institute of Health's web-based training on "Protecting Human Research Participants".

Participants consisted of a sample of 354 male and female volunteers ages 18 to 64 years, living with HIV, and attending any of the nine comprehensive care and treatment centers under the umbrella of Nazareth Hospital, in Nairobi, Kenya. According to Kenya's Epidemiological Fact Sheet on HIV and AIDS, most of the people infected with HIV are within the 18 to 64 year-old age bracket (WHO, 2008). Cases with missing values were deleted, leaving 354 who completed the survey and were included in the study. This sample size was adequate (Cohen, 1988). 


\section{Inclusion and Exclusion Criteria}

Participants included an equitable (unbiased) sample of men and women living with HIV who were 1) at least 18 years old (the legal age at which a person can give informed consent in Kenya), 2) able to communicate in either Kiswahili or English, and 3) attending any of the nine HIV treatment clinics of Nazareth Hospital in Nairobi, Kenya. Individuals who were 1) under 18 years of age, 2) prisoners, 3) pregnant, or 4) those with diminished autonomy because of their inability or limitation to give informed consent. To ensure the anonymity and confidentiality of the participants, personal identifiers, such as name, addresses, telephone number, and birth date were not recorded on any of the survey tools and the administration of the surveys was carried out in private rooms. In addition, research data were kept separate from identifiable information, such as the informed consent forms of the participants.

\section{Instrumentation}

The demographic characteristics survey was used to gather information on participants' gender, age, education, employment status, marital status, and religion. To understand the composition of the sample, participants were classified into four age groups (18-30, 31-40, 41-50, and 51-64), four religious groups (Catholic, Protestant, Muslim, and traditional African), and five marital status groups (never married, married, separated, divorced, and widowed).

The Interpersonal Support Evaluation List (ISEL) was used to collect data on perceived social support (Cohen \& Hoberman, 1983; Cohen \& Wills, 1985). The ISEL is designed to capture individuals' perceptions about availability of social support if and when it is needed. Research has shown that perceived support is more important than received support in individuals' adjustments to stressful life events (Wethington \& Kessler, 1986); therefore, it is an appropriate measure in this study. Items are counterbalanced for social desirability, with 20 reverse-coded Likert-type statements designed to assess four separate types of social support - emotional, informational, material, and belonging. Each of these types of support has 10 items. The psychometric properties of the ISEL are well established. The validity of the ISEL and other social support measures ranges from .30 to .46 , whereas the reliability of the overall alpha coefficients ranges from .88 to .90 .

The survey tool for CSE consisted of 26 items that measured an individual's belief that he or she can perform behaviors important to adaptive coping by sorting out what is controllable and what is uncontrollable (Chesney, Chambers, Taylor, Johnson, \& Folkman, 2003; Chesney et al., 2006). Participants were asked to indicate on a scale from 0 to 10 ( 0 - cannot do at all, 5 - moderately certain I can do, and 10 - certain I can do) how confident they are that they can do certain things when their situation is not good. For example, "When things are not going well with you, how confident are you that you can talk positively to yourself/sort out what can be changed and what cannot be changed/get emotional support from friends and family?" (Chesney et al., 2003; Chesney et al. 2006). An overall CSE score was created by summing the item ratings $(\alpha=.95$; scale mean $=137.4, S D=45.6)$ (Chesney et al., 2003, 2006). In this study, Cronbach alpha coefficients were 0.905 for the ISEL and 0.860 for CSE.

\section{RESULTS}

Descriptive statistics were used to summarize data (Portney \& Watkins, 2000). The majority of the participants were women $(n=253,71.47 \%)$. The largest proportion of participants were between 31 and 40 years of age $(43.5 \%)$ and were women $(45.5 \%)$.

Most of the participants were married $(56.5 \%)$ or widowed (16.9\%). More men were married (74\%) than women (49\%); more women were widowed (19\%) than men (11\%); more women were separated (13.6\%) than men $(5.7 \%)$; and only $4.7 \%$ of all participants were divorced.

Most of the participants had completed only primary education (42.1\%) or secondary education (38.4\%). More men had a high school education (50.4\%) than women (33.6\%). Most men and women $(71.2 \%)$ were engaged in some form of economic activity (formal, informal, or farming sectors). More men were employed in the formal sector $(40.6 \%)$ than women $(32.2 \%)$ and $28 \%$ of both men and women were unemployed. 
Standard multiple regression was performed to test three hypotheses: 1) the relationship between demographic characteristics and coping self-efficacy, 2) the relationship between coping self-efficacy (CSE) and the composite (undimensional) social support, and 3) the relationship between CSE and the four dimensions of social support - Material, Emotional, Informational, and Belonging. Demographic characteristics were used as control variables. Overall demographic characteristics did significantly predict Coping Self-Efficacy $(\mathrm{F}(6,354)=1.09$, P> .406)

In the second regression, the $F$ test for the overall model - which included composite social support-was significantly different from zero, with $F(5,354)=10.316,<.0001$. The model's $\mathrm{R} 2$ was $=.132$, indicating that these variables, as a set, explain about $13.2 \%$ of variance in CSE. Table 1 displays the results of the evaluation of the contribution and significance of each of the variables in the model. The results reveal that the coefficient of social support significantly predicts CSE, $t=9.563,<.000$. A coefficient for the variable ISEL $=.298$ indicates that for every unit increase in social support, CSE would increase by .298 units, with a confidence interval (CI) of .210.386 .

Then the third standard multiple regression was performed between CSE as the outcome variable and all four dimensions of social support - emotional, informational, material, and belonging - entered in the model simultaneously as predictor variables. $R$ for the regression was significantly different from zero, $F(4,354)=13.472$, $p<.001$. As a set, the dimensions of social support predict CSE, which account for about $14 \%$ of variation in CSE. Further, upon examination of the contribution and significance of each of these dimensions of social support, while controlling for the effect of others, two dimensions significantly predicted CSE - emotional support $\mathrm{t}=2.779, p=$ .003 , and informational support $\mathrm{t}=2.413, p=016$. Material and belonging support were not significant.

\section{DISCUSSION}

The findings of this study can be explained by integrating the stress, appraisal, and coping theory of Lazarus and Folkman (1984) and Bandura's theory of perceived self-efficacy (Bandura, 1997) to show how the social environment can shape the thoughts of people living with HIV. As Lazarus and Folkman theorize, the findings in this study appear to support the dynamic transaction or the interplay between personal and social environmental variables. In this regard, individuals who perceive that social support is available are also likely to report a strong belief in their capability to protect themselves from being psychologically harmed and to combat HIV-related or unrelated stressors.

\section{Social Support (Composite) and CSE}

The data from the multiple regressions infer that men and women living with HIV, who perceive that social support (composite) is available, are also likely to believe in their ability to find meaning in negative events by focusing on the positive side, engaging in activities that can impede unpleasant thoughts and emotions, and seeking support from family and friends.

Clearly, stressor response variability among people living with HIV, and under comparable conditions, may be determined by an individual's perception of his or her ability to do something than can mitigate the potential pathogenic effects of the negative events. Whether people believe they can perform specific tasks depends upon their perception of available support from either themselves or their social network (Cohen \& Hoberman, 1983; Cohen \& Wills, 1985). Our determination of an association between an individual's perceptions of social support and CSE implies that the social network used by Kenyans living with HIV is generating interpersonal resources that are responsive to the needs elicited by stressful events. The significant relationship found between social support and CSE in this study is consistent with other studies that have established a relationship between social support and mental health status. People's perceived lack of social support has been found to be a significant predictor of low self-efficacy (Simoni et al., 2002) whereas social support has shown to maintain and improve the overall functioning of older adults with chronic obstructive pulmonary disease (Sirey, Raue, Alexopoulos, 2008). Further, studies focusing on people living with HIV have found significant relationships between social support and a positive state of mind (Gonzalez et al., 2004) and social support with self-efficacy (Chesney et al., 2000). 
Our findings support the notion that the large healthcare provider from which this sample was drawn was able to mobilize social support from existing networks and provide other psychosocial interventions to people living with HIV that positively affected their abilities to cope with life stressors. The interventions provided by this healthcare system included training sessions, individual and group counseling, and nesting in support groups determined by patient categories, such as discordant couples, youth, widows or widowers, and even children support groups. During the regular meetings, the groups shared experiences and the individuals encouraged one another. This social and interpersonal sharing of common experiences of people living with HIV is likely to have created opportunities for vicarious and persuasive experiences, which are essential building blocks of CSE. It is possible that the observance of their peers' success in fighting stressors enhanced their belief that men and women living with HIV have the capability to do the same. Conversely, if other members nested in a social network with people who are unsuccessfully fighting life stressors, they may convince themselves that they are not proficient to take on similar situations.

\section{Dimensions of Social Support and CSE}

The findings also show that men and women living with HIV, who perceive that emotional, informational, and belonging (dimensions of) support are available, are also likely to believe in their ability to regulate emotions, manage problems, and seek social support from friends, family, and community. A model with all four dimensions of support accounted for approximately $14 \%$ of the variation in CSE.

Our findings of significant relationships between two of the four dimensions of social support (i.e., emotional and informational) suggest that people living with HIV need an array of support resources to lessen the potential effects of situational stressful factors. The experience of being loved despite one's shortcomings or weaknesses, having confidants, family members, friends, or a social group, may assist people under stress to engage in activities that distract them from negative thoughts of past, present, or future experiences (Cohen and Wills, 1985). Additionally, informational support may engender beliefs in individuals' capabilities as it fosters transfer of credible, trustworthy, and critical information that these individuals need to understand and solve problems.

\section{Comprehensive HIV Treatment and Care}

In the present study, psychosocial support provided in various ways - telephone calls, home visits, community services, provision of materials and information, and individual or group counseling among participants - could empower belief in the capability to cope with life stressors. Regarding belonging support, participants indicated that they had at least one person (a confidant) with whom they could meet and spend time enjoying common things, which could reduce feelings of loneliness and isolation arising from stigma and discrimination. Participants had at least one person whose advice they could trust or whose suggestions on how to deal with personal or family problems they could depend upon.

Participants may have engaged in this form of coping to deal directly with problems causing emotional or psychological distress. Their belief in their ability to engage in strategies to reduce or eliminate the problem - e.g., "find solutions to your most difficult problems" or "break an upsetting problem down into smaller parts" (Chesney et al., 2003) - may have positively influenced strategies used to solve their problems. Their belief that they can manage problems may have fostered strategies of coping whereby they carefully consider various alternatives, weigh them, make choices among them, and act upon their preferences (Lazarus \& Folkman, 1984).

Consistent with the Lazarus and Folkman (1984) theory on problem-focused forms of coping, data from this study demonstrated that participants believed in their ability to engage with inward or outward strategies to deal with stressors. Their belief in their ability to tap into external resources and seek help from friends, family, and the community to cope with problems (e.g., "get friends to help you with things you need", "get emotional support from friends/family", or "get emotional support from community organizations") may be rooted in their perception that support would be available if required. 


\section{LIMITATIONS}

As with all studies, this study presented design issues that affect the generalizability of its findings. First, this study used a cross-sectional design to investigate adherence to HIV medication at only one point in time. A cross-sectional study design may not have detected changes in this type of social support needed to attenuate the effects of stressors. Furthermore, no cause and effect can be inferred because the study was not controlled and randomized; therefore, the results do not suggest the direction of influences. It is possible that individuals who possess a stronger sense of CSE perceive social support to be available rather than vice versa. This study also used a convenience sample of willing patients who were prescribed HIV medication and were being served in one of the nine comprehensive care and treatment centers under the umbrella of a major healthcare provider. This sampling method limits the degree to which the findings can be generalized to other populations in the Central Province and the metropolitan city of Nairobi, as well as the rest of Kenya or Africa. To make the findings generalizable, it is essential to randomize the sample selection and include different locations and more providers of HIV treatment and care centers because of the differing quality of services. Although the sampling method used can be considered a limitation in the study design, the sample was reflective of the national demographic characteristics of Kenya, which can lead one to infer that the results from the sample may be reliable despite the fact that a convenient sampling method was used.

To ensure greater generalizability and applicability of findings, future investigations should utilize a longitudinal study design across diverse regions and healthcare facilities, thereby enabling researchers the opportunity to assess the role of social support in influencing CSE in a diverse population of subjects over time. In addition, designing future studies to assess what factors may trigger emotional or psychological distress and to evaluate the effectiveness of resources available for patients to use to cope with such stressors would assist in expanding the evidenced-based knowledge.

\section{CONCLUSION}

In conclusion, clearly, one of the most effective ways to develop coping self-efficacy to deal with HIVrelated stress among people living with HIV is to engage families and communities to create and strengthen a supportive social environment. Although people can be trained to be self-efficacious in dealing with difficult situations of life, it is critical to provide them with social support. As such, designers of HIV coping programs need to consider social support as an essential resource for coping self-efficacy. However, the challenge is how to improve social environment to generate these resources.

\section{AUTHOR INFORMATION}

Dr. Thomas Matenjwa Kamau is Assistant Professor of Health Sciences of Ohio University, Zanesville. He received his PhD from School of Health and Medical Sciences at Seton Hall University, New Jersey. He also holds two masters; MBA concentration in Accounting, and MPA with a concentration in Healthcare Policy and Management. He teaches courses in health services administration and community health such as the Introduction to Public health, Human Resource Management, Administration of Acute Care Facility. His current research interests include health policy and management, HIV prevention, treatment and management of chronic and communicable diseases in the developing countries. E-mail: kamau@ohio.edu (Corresponding author)

Dr. Valerie G. Olson, Associate Professor, serves as Program Director of the Doctor of Physical Therapy Programs at Dominican College. She earned her PhD from Seton Hall University in 2000. In addition to providing the leadership to the doctoral programs, Dr. Olson's scholarship consists of teaching and research in teaching and learning, faculty professional development, and curriculum design in higher education as well as in health professions education. In addition, Dr. Olson focuses on a variety of issues and trends in health care, such as cultural diversity, algorithmic decision making, and interdisciplinary approaches to the management of the neurologically involved child and adult. E-mail: valerie.olson@dc.edu

Dr. Genevieve Pinto Zipp is Associate Professor in the Department of Graduate Programs in Health Sciences at Seton Hall University. She received her EdD from Teachers College Columbia University in 1996. She currently 
teaches management of neuromuscular problems in the Doctor of Physical Therapy program. Her research interests focus on, a) effects of performing dual tasks on walking performance and postural sway in children and adults, and b) curriculum design issues including the use of mind mapping and video based cases in professional education for the promotion of clinical decision making skills. E-mail: genevieve.zipp@shu.edu

Dr. Mary Ann Clark, EdD is professor and dean, School of Health Professions and Nursing, CW Post Campus, Long Island University. She received her EdD in Higher Education Administration and Management from University of Houston. Her current research interests include assessment of childhood obesity, teaching strategies, and higher education assessment and accreditation. E-mail: maryann.clark@liu.edu

\section{REFERENCES}

1. Bandura, A. (1997). Self-efficacy: The exercise of control. New York: WH. Freeman.

2. Catz, SL, Kelly, JA., Bogart, LM, Benotsch, EG, McAulife, TL. (2000). Patterns, correlates, and barriers to medication adherence among persons prescribed new treatments for HIV disease. Health Psychology, 19 (2):124-133

3. Central Bureau of Statistics. (2004). Kenya Demographic and Health Survey 2003. Nairobi, Kenya: Ministry of Health.

4. Chesney, MA., Chambers, D B., Taylor, JM., Johnson, LS., \& Folkman, S. (2003). Coping effectiveness training for men living with HIV: Results from a randomized clinical trial testing a group-based intervention. Psychosomatic Medicine, 65(6), 1038-1046.

5. Chesney, MA., Ickovics, JR., Chambers, DB., Gifford, AL., Neidig, J., Zwickl, B. \& Wu, AW. (2000).Selfreported adherence to antiretroviral medications among participants in HIV clinical trials: The AACTG Adherence Instruments. AIDS Care, 12(3), 255-266.

6. Chesney, MA., Neilands, TB., Chambers, DB., Taylor, JM., \& Folkman, S. (2006). A validity and reliability study of the coping self-efficacy scale. British Journal of Health Psychology, 11(3), 421-437.

7. Cohen, J. (1988). Statistical power analysis for the behavioral sciences (2nd Ed.). Hillsdale, N.J: Erlbaum.

8. Cohen, S., \& Hoberman, H. (1983). Positive events and social supports as buffers of life change stress. Journal of Applied Psychology, 13(2), 99-125.

9. Cohen, S., Janicki-Deverts, D., \& Miller, GE. (2007). Psychological stress and disease. The Journal of the American Medical Association [JAMA], 298(14), 1685-1687.

10. Cohen, S., \& Wills, TA. (1985). Stress, social support, and the buffering hypothesis. Psychological Bulletin, 98(2), 310-357.

11. Gonzalez, JS., Penedo, FJ., Antoni, M., Durán, RE., McPherson-Baker, S., Ironson, G., \& Schneiderman, N. (2004). Social support, positive states of mind, and HIV treatment adherence in men and women living with HIV/AIDS. Health Psychology, 23(4), 413-418.

12. Hutchinson, C. (1999). Social support: Factors to consider when designing studies that measure social support. Journal of Advanced Nursing, 29(6), 1520-1526.

13. Langford, CPH., Bowsher, J., Maloney, JP., \& Lillies, PP. (1997). Social support: A conceptual analysis. Journal of Advanced Nursing, 25(1), 95-100.

14. Lazarus, RS., \& Folkman, S. (1984). Stress, appraisal, and coping. New York: Springer.

15. Leserman, J., Petitto, JM., Gu, H., et al. (2002). Progression to AIDS, a clinical AIDS condition and mortality: Psychosocial and physiological predictors. Psychological Medicine, 32, 1059-1073.

16. Marino, P., Sirey, JA., Raue, PJ., Alexopoulos, GS. (2008). Impact of social support and self-efficacy on functioning in depressed older adults with chronic obstructive pulmonary disease (2008). International Journal of Chronic Obstructive Pulmonary Disease, 3(4), 713 - 718.

17. Munro, BH. (2005). Statistical methods for health care research (5th Ed.). New York: Lippincott Williams \& Wilkins.

18. Portney, LG., \& Watkins, MP. (2000). Foundations of clinical research: Applications to practice. Upper Saddle River, NJ: Prentice Hall.

19. Schwarzer, R., \& Renner, B. (2000). Social-cognitive predictors of health behavior: Action self-efficacy and coping self-efficacy. Health Psychology, 19(5), 487-495.

20. Siegel, K., \& Scrimshaw, E. W. (2005). Stress, appraisal, and coping: A comparison of HIV-infected women in the pre-HAART and HAART eras. Journal of Psychosomatic Research, 58(3), 225-233. 
21. Tabachnick, BG. \& Fidell, LS. (2001). Using Multivariate Statistics, (4th, Ed.). Boston, MA: Allyn and Bacon.

22. Theuninck, A C., Lake, N., \& Gibson, S. (2010). HIV-related posttraumatic stress disorder: Investigating the traumatic events. AIDS Patient Care and STDs, 24(8), 485-491.

23. Wethington, E., \& Kessler, R C. (1986). Perceived support, received support, and adjustment to stressful life events. Journal of Health and Social Behavior, 27(1), 78-89.

24. World Health Organization. (2008, February 18). Epidemiological fact sheet on HIV and AIDS:Core data on epidemiology and response. World Health Organization. Retrieved October 11, 2011, from World Health Organization Web site:

http://apps.who.int/globalatlas/predefinedReports/EFS2008/full/EFS2008_KE.pdf 
NOTES 